\title{
Children with Specific Language Impairment also show impairment of music-syntactic processing
}

\author{
Sebastian Jentschke, ${ }^{1 *}$ Stefan Koelsch, ${ }^{1,2}$ Stephan Sallat, ${ }^{3}$ \& Angela D. Friederici ${ }^{1}$ \\ ${ }^{1}$ Max Planck Institute for Human Cognitive and Brain Sciences, Leipzig, Germany \\ ${ }^{2}$ University of Sussex, Brighton, UK \\ ${ }^{3}$ Justus Liebig University, Giessen, Germany \\ * To whom correspondence should be addressed: \\ Max Planck Institute for Human Cognitive and Brain Sciences, Stephanstr. 1A, \\ 04103 Leipzig, Germany, jentschke@cbs.mpg.de
}

Both language and music consist of sequences that are structured according to syntactic regularities. We used two specific event-related brain potential (ERP) components to investigate music-syntactic processing in children: the ERAN (Early Right Anterior Negativity) and the N5. The neural resources underlying these processes have been posited to overlap with those involved in the processing of linguistic syntax. Thus, we expected children with specific language impairment (SLI, which is characterized by deficient processing of linguistic syntax) to demonstrate difficulties with music-syntactic processing. Such difficulties were indeed observed in the neural correlates of music-syntactic processing: neither an ERAN nor an N5 was elicited in children with SLI, whereas both components were evoked in age-matched control children with typical language development. Moreover, the amplitudes of ERAN and N5 were correlated with subtests of a language development test. These data provide evidence for a strong interrelation between the language and the music processing system, thereby setting the ground for possible effects of musical training in SLI therapy.

\section{Introduction}

Music and language provide two examples of highly structured systems to which we are exposed in everyday life. Both consist of perceptually discrete elements, organized in hierarchically structured sequences (see, e.g., Deutsch, 1999; Lerdahl, 2001). The combination of these structural elements into sequences is governed by sets of principles, commonly denoted as syntax (see, e.g., Koelsch, 2005; Patel, 2003; Riemann, 1877). The human brain internalises syntactic regularities by mere exposure, and the acquired implicit knowledge of such regularities influences perception and performance (see McMullen \& Saffran, 2004; Tillmann, Bharucha, \& Bigand, 2000).

We used a chord sequence paradigm suited to elicit two electric brain responses reflecting music-syntactic processing: An early right anterior negativity (ERAN) and a late negativity (N5) (Koelsch, Gunter, Friederici, \& Schröger, 2000; Koelsch et al., 2001; Leino, Brattico, Tervaniemi, \& Vuust, 2007; Loui, Grent-'t-Jong,
Torpey, \& Woldorff, 2005). The ERAN is assumed to reflect early and fairly automatic processes of syntactic structure building (Koelsch, 2005; Koelsch, Schröger, \& Gunter, 2002). Its amplitude size can be modulated, e.g. by musical training (Koelsch, Schmidt, \& Kansok, 2002). It is typically followed by an N5 (a negativity maximal around $500 \mathrm{~ms}$ ), which has been posited to reflect processes of harmonic integration (Koelsch, 2005; Koelsch et al., 2000). Both ERPs can be elicited in 5-year old children (Koelsch et al., 2003), and recent data indicate that they can even be observed even in $2 \frac{1}{2}$ year-olds (Jentschke, 2007).

During the last years, a number of studies has shown that processing of both musical and linguistic syntax relies on overlapping cognitive resources (Koelsch, Gunter, Wittfoth, \& Sammler, 2005; Patel, Gibson, Ratner, Besson, \& Holcomb, 1998; Slevc, Rosenberg, \& Patel, 2007), some of which are located in overlapping brain areas (such as lateral parts of the inferior frontal gyrus and the anterior superior temporal gyrus; Koelsch, 2005; Patel, 2003). Moreover, 
substantial evidence underlines the importance of a sophisticated processing of prosody (i.e., the "musical" features of speech) for the acquisition of language (Fernald, 1989; Jusczyk, 2002; Jusczyk et al., 1992; Krumhansl \& Jusczyk, 1990). In accordance, several studies reported a relationship between musical and prosodic abilities (Magne, Schön, \& Besson, 2006; Schön, Magne, \& Besson, 2004), as well as between musical and phonological abilities (Anvari, Trainor, Woodside, \& Levy, 2002; Slevc \& Miyake, 2006; Wong, Skoe, Russo, Dees, \& Kraus, 2007).

The present study aimed at investigating music processing in children with Specific Language Impairment (SLI). These children (about $7 \%$ of the population, slightly more males) have linguistic difficulties in the absence of possible explanatory factors that usually accompany language impairment (i.e., deficiencies in intelligence, hearing, oral motor function, etc.; for a review, see, e.g., Leonard, 2000). They acquire language not as rapidly and effortlessly as other children and may be protracted in their development of language perception and production. A main characteristic of them is that they show severe difficulties with grammar (van der Lely, 2005). They perform worse on many measures of syntactic comprehension, especially those concerning syntactic complexity (Botting, Faragher, Simkin, Knox, \& Conti-Ramsden, 2001; Marinellie, 2004). In general, it seems that lexical and pragmatic skills are relatively intact, with phonology and argument structure abilities being slightly worse, and morphosyntactic skills (particularly processing of grammatical morphemes) being most impaired. So far, very few studies investigated music perception in children with language disorders (Alcock, Passingham, Watkins, \& Vargha-Khadem, 2000; Overy, Nicolson, Fawcett, \& Clarke, 2003) and, to our knowledge, none so far in children with SLI. ${ }^{1}$

The present study will fill in this gap by investigating music processing, specifically the processing of music-syntactic regularities, in children with SLI. Given the reported overlap in the neural networks for language and music, we hypothesized that children with SLI (having deficiencies in the processing of linguistic syntax) will also have difficulties in the processing of musical syntax.

1 Bishop and McArthur (2004) measured ERPs to tone pairs and single tones but not to musical phrases (see also McArthur \& Bishop, 2004, 2005).

\section{Materials and Methods}

Participants. Two groups of children with either typical language development (TLD) or specific language impairment (SLI) were compared. All children were right-handed and native speakers of German. Their parents gave written informed consent.

Data of the children with SLI $(N=21)$ were acquired at a kindergarten for special education where they were treated to improve their language skills. Before entering the kindergarten, the children were screened for intelligence, language abilities, normal hearing and neurological deficits by a public health officer and speech therapists. Only children for which parents and teachers reported normal hearing and no history of hearing disease were included. Datasets were excluded from the analysis, if [1] the EEG measurement could not be evaluated, [2] the amplitudes of their ERP responses were outliers with respect to the distribution of both groups (see Statistical evaluation), [3] the children had less than $70 \mathrm{IQ}$ points in the nonverbal part of the Kaufman Assessment Battery for Children (Kaufman, Kaufman, \& Melchers, $1994),{ }^{2}$ or [4] they were not at least 1.5 SD below the mean of the population in any subtest of a language screening (Grimm, Aktas, \& Frevert, 2001). All in all, the data of 15 children with SLI were evaluated ( $4 ; 8$ to $5 ; 11$ years old, $M=5 ; 2$ years; 9 boys, 6 girls).

The children of the TLD group $(N=24)$ were recruited from public kindergartens in Leipzig. The same criteria for inclusion and exclusion were applied, however, none of these children had to be excluded because of criterion [3], or a score more than $1 \mathrm{SD}$ below the population mean in any subtest of the language development test. In this group, the data of 20 children were evaluated (4;3 to $5 ; 11$ years old, $M=5 ; 3$ years; 10 boys, 10 girls).

2 Decreased intelligence is regarded as risk factor, but most authors agree that an IQ between 70 and 85 points cannot be an exclusive explanation for language impairment (for a discussion, see Bishop, 2004; Botting, 2005; Leonard, 1998). Some researchers argue in favour of a further criterion: values in any subtest of the language screening should be more than 1.0 SD below the nonverbal IQ. A separate analysis was conducted in which children who did fulfil this criterion (1 girl and 1 boy) were temporarily excluded. Since the pattern of results was similar, these children were re-included to improve the statistical power of the analyses. 


\begin{tabular}{lrrrrrr}
\hline & & \multicolumn{3}{c}{ socioeconomic status } & \multicolumn{3}{c}{ duration of education } & nonverbal \\
& & mother & father & mother & father & IQ \\
Children... & $N$ & 28 & 26 & 25 & 22 & 34 \\
\cline { 3 - 7 } with SLI & $M(S E M)$ & $34.55(3.79)$ & $38.22(5.11)$ & $12.82(0.60)$ & $12.13(1.16)$ & $85.29(1.95)$ \\
with TLD & $M(S E M)$ & $55.82(3.72)$ & $50.94(5.21)$ & $16.00(1.13)$ & $16.14(1.27)$ & $107.25(2.25)$ \\
\hline
\end{tabular}

Table 1. Group comparision of the mean and standard error of mean (in parantheses) of the socioeconomic status of the parents' eduction, their duration of education (in years), and the non-verbal IQ of the children.

ERP responses to the onset of the first chord of the sequence did not differ between groups, indicating that the two groups did not differ in their hearing capabilities and their processing of acoustic features (see Results section). Although children were excluded if their intelligence was not within a normal range, the nonverbal intelligence in the children with SLI was lower than in the children with TLD $\left(t_{(32)}=6.97\right.$; $p>0.001$; see Table 1). Similar differences were observed for the parents' duration of education (in years) which was shorter for the parents of children with SLI (mothers: $t_{(23)}=$ 2.29; $p=0.032$; fathers: $t_{(20)}=2.11 ; p=0.047$ ), and the socioeconomic status of their occupation (ISEI) which was also lower in this group (mothers: $t_{(26)}=3.84 ; p=0.001$; fathers: $t_{(21.6)}=$ $1.74 ; p=0.096) .^{3}$ However, as will be reported in the Results section, the education, and socioeconomic status of the parents were not related with the investigated ERP components.

Behavioural measures. In further sessions (each approx. $1 / 2$ to 1 hour), we obtained the current status of the linguistic development, the non-verbal intelligence, and the musical skills of the participants.

Linguistic skills were evaluated with a standardized language development test (SETK 35; Grimm et al., 2001) which consisted of four subtests evaluating different aspects of language processing:4 [1] "Sentence comprehen-

3 To determine the socio-economic background of the children's families, the occupation of the parents was classified in terms of the "International Standard Classification of Occupation 1988" (ISCO-88; International Labour Organization, 1990). This classification was transformed into "International Socio-Economic Index of Occupational Status" values (ISEI; Ganzeboom \& Treiman, 1996) to provide a status measure for this occupation.

4 The population norms for these subtests were national norms based on 495 children from 11 age groups ( $3 ; 0$ to $5 ; 11$ years; each a half year wide) that were equally distributed with regard to sex and age group (see Grimm et al., 2001 for a more detailed description). For a further, fifth subtest - "Memory for Words" - no population sion" reflecting the complex interplay of phonologic, lexical-semantic, and morphologicsyntactic processing steps. [2] "Generation of plurals" being related to syntactic processing, especially knowledge of morphological rules. Children with SLI are hampered in their extraction of such rule-based patterns from spoken language. [3] "Non-word repetition" being a measure of the ability to process and to store unknown phoneme patterns in short-term memory. Difficulties in this subtest are considered a classical marker of SLI. [4] "Repetition of sentences" reflecting grammatical knowledge and working memory functions, i.e. the ability to employ knowledge of grammatical structures in order to process sentences and to store them in memory in a compact form.

Non-verbal intelligence was tested with the Kaufman Assessment Battery for Children (Kaufman et al., 1994). The task sets differed slightly between the 4-year-olds and the 5year-olds and contained the subtests "Hand Movements" (repeating a sequence of hand movements), "Triangles" (constructing a given figure with rubber triangles), and "Spatial Memory" (remembering the position of objects; only for 5 -year-olds). ${ }^{5}$

Musical skills were measured with selfauthored tests. ${ }^{6}$ From these tests factors were

norms are provided. Therefore, results of this subtest were not evaluated.

5 The population norms were gained from a national sample with 3,098 children from 40 age groups ( $2 ; 6$ to $12 ; 5$ years; each 3 months wide). The sample was representative with regard to the level of education of the parents, and equally distributed with regard to sex and age group. Two further subtests were not evaluated: "Gestalt Closure" (determining which complex figure differs from other figures in a set; only for 5-year-olds) did not conform to a standard normal distribution and for "Face Recognition" (recognizing a particular face within a picture; only for 4-year-olds) only few measurements were acquired.

6 Only 25 of all children who were measured with EEG participated in these tests of musical abilities (all subjects of the SLI group and 10 subjects of the TLD group). 
extracted (using principal component analysis), accounting for different classes of musical abilities (e.g., memory for musical phrases, reproduction of rhythms, etc.). Only one of these factors ("Memory for Musical Phrases") was significantly correlated with the amplitude of the ERP components (see Results). In the tasks, underlying this factor, the children determined (in a paired comparison), if they heard an original phrase again or a melodically or rhythmically modified version of this phrase. The original musical phrases were either (wellknown) beginnings of German children songs or (unknown) self-composed melodies (of 3 to 4 beats length). Reaction times and the proportion of correct responses were measured. The factor was extracted from the reaction time measures and reflects the abilities of melodic and rhythmic-melodic processing as well as of storing musical phrases in memory. Skills that were not correlated with the ERP measures (see also Discussion), included tasks that were related to long term memory for musical material (e.g., to indicate on a picture, showing the main themes of four well-known songs, which song was played) or music production (e.g., to sing back a song or to produce ones' favourite song).

Sequences were transposed to the 12 major keys, resulting in 24 different sequences. All were played with a piano sound with the same decay of loudness for all chords (generated using Steinberg Cubase SX and The Grand; Steinberg Media Technologies, Hamburg, Germany). Both sequence types were randomly intermixed (with a probability of 0.5 for each sequence type) and presented in direct succession (Figure 1C). Moreover, each sequence was presented pseudo-randomly in a tonal key different from the key of the preceding sequence. Across the experiment, each sequence type was presented eight times in each of the 12 major keys, resulting in 192 sequences for the entire experiment. During the experiment (approx. $17 \mathrm{~min}$ ) children sat in front of a monitor and saw a silent movie of an aquarium.
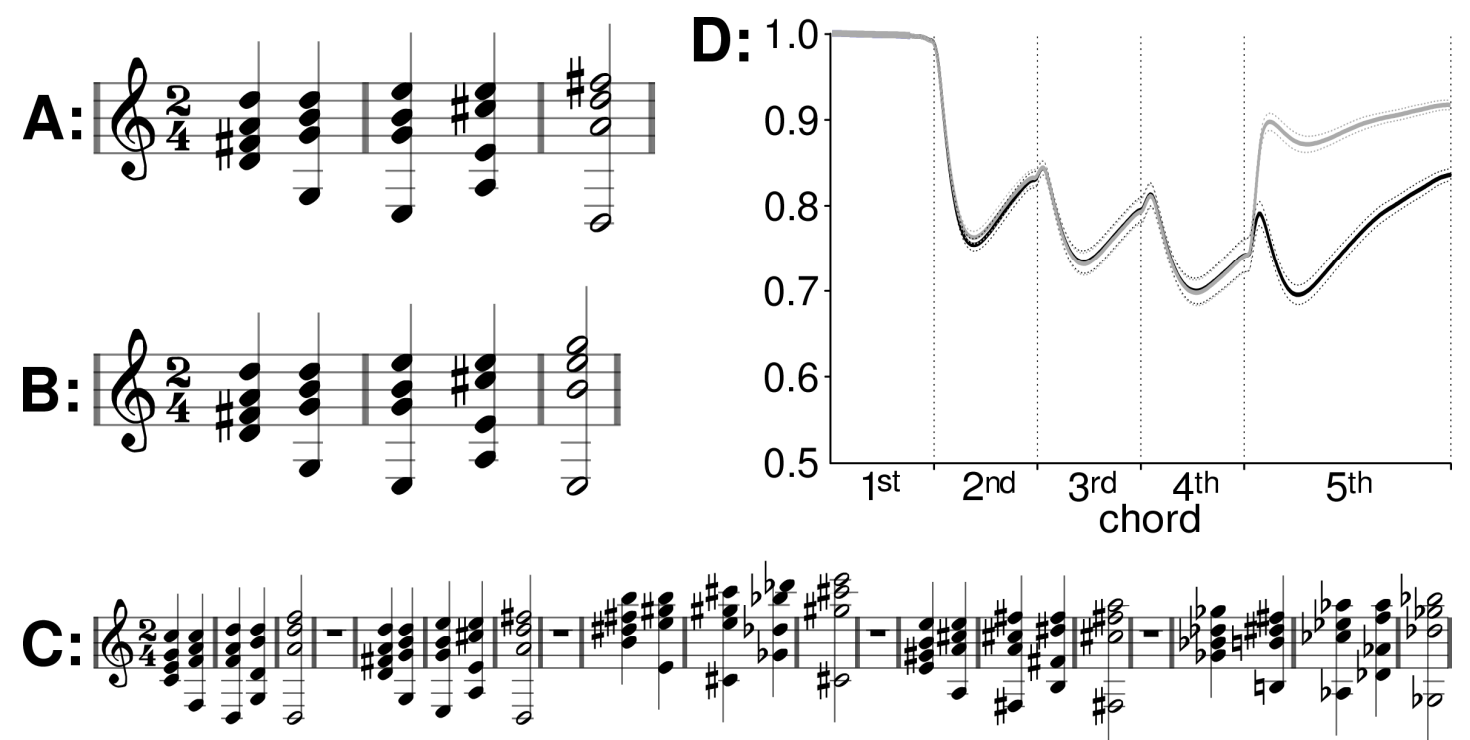

Figure 1. Chord sequences used in the experiment either ending on a regular tonic (A) or an irregular supertonic (B). In the experiment, chord sequences were played in direct succession $(\mathrm{C}$, solid arrows indicate tonics, and dotted arrows supertonics). (D) shows the correlation of the local context (pitch representation of the current chord) with the global context (pitch representation of the previous chords stored in echoic memory). Note that the music-syntactically irregular supertonics (grey line) were even more congruent with the information stored in the echoic memory than the regular tonic chords (black line). Each line represents the mean of all twelve major keys (small dotted lines represent standard error of mean). The modelling was performed using the IPEM Toolbox (Leman, Lesaffre, \& Tanghe, 2005). 
EEG recording and processing. EEG data were recorded with $\mathrm{Ag}-\mathrm{AgCl}$ electrodes from 22 scalp locations - FP1, FP2, F7, F3, FZ, F4, F8, FC3, FC4, T7, C3, CZ, C4, T8, CP5, CP6, P7, P3, PZ, P4, P8, O1, O2 according to the Extended International 10-20 System (American Electroencephalographic Society, 1994) - and 5 further locations on the head nose tip, outer canthi of both eyes, left (M1) and right mastoid (M2). Data were sampled at $250 \mathrm{~Hz}$, with a reference at the left mastoid and without online filtering using a PORTI32/MREFA amplifier (TMS International B.V., Enschede, NL). Impedances of the scalp electrodes were kept below $3 \mathrm{k} \Omega$, of the head electrodes below $10 \mathrm{k} \Omega$. Data were processed offline using EEGLab 4.515 (Delorme \& Makeig, 2004). The data were re-referenced to linked mastoids (mean of M1 and M2), filtered with a $0.25 \mathrm{~Hz}$ high-pass filter (finite impulse response (FIR), 1311 pts; to remove drifts) and a $49-51 \mathrm{~Hz}$ band-stop filter (FIR, 437 pts; to remove line noise). Then an independent component analysis (ICA) was conducted and artefact components (e.g., eye blinks, eye movements, or muscle artefacts) were removed. Thereafter, data were rejected for [1] threshold (if amplitudes exceeded $\pm 120 \square \mathrm{V}$ ), [2] linear trends (if linear trends exceeded $160 \square \mathrm{V}$ in a 400 ms gliding time window), [3] improbable data (if the trial was lying outside a $\pm 6 S D$ range (for a single channel) or $\pm 3 \mathrm{SD}$ range (for all channels) of the mean probability distribution), ${ }^{7}$ [4] abnormally distributed data (if the data were lying outside a $\pm 6 S D$ range (for a single channel) or a $\pm 3 \mathrm{SD}$ range (for all channels) of the mean distribution of kurtosis values), ${ }^{8}$ and [5] improbable spectra (spectra should not deviate from the baseline spectrum by $\pm 30 \mathrm{~dB}$ in the 0 to $2 \mathrm{~Hz}$ frequency window [to reject eye movements] and $+15 /-30 \mathrm{~dB}$ in the 8 to $12 \mathrm{~Hz}$ frequency window [to reject alpha activity]). Finally, non-rejected epochs ( $M$ = 60) were averaged for a period of $200 \mathrm{~ms}$ before (baseline) to $1200 \mathrm{~ms}$ after stimulus onset (length of the final chord).

Statistical evaluation. For statistical evaluation of ERPs, four regions of interest (ROls) were computed (see schematic head in Figure 3): left-anterior (F3, F7, FC3), right-anterior (F4, F8, FC4), left-posterior (P3, T7, CP5), and right-posterior (P4, T8, CP6). To ensure that

7 It is assumed that trials containing artefacts are improbable events.

8 It is assumed that data epochs with artefacts sometimes have very "peaky" activity value distributions resulting in a high kurtosis whereas abnormal flat epochs have a small kurtosis. both groups did not differ in their processing of acoustic features, ERPs elicited by the chords at the first position of the sequences were evaluated. In this analysis, the mean amplitude values in two time windows were used (relative to stimulus onset): [1] 0 to $100 \mathrm{~ms}$, and [2] 100 to $200 \mathrm{~ms}$. Mixed-model ANOVAs for repeated measurements containing the within-subject factors time window $(0$ to $100 \mathrm{~ms} v \mathrm{vs} .100$ to $200 \mathrm{~ms}$ ), anterior-posterior distribution, and hemisphere (left vs. right) and the betweensubjects factor group (TLD vs. SLI) were performed for statistical evaluation (see Results for details). For the comparison of the ERP response to the irregular supertonics and the regular tonics, the mean amplitude values in four time windows, centred on the peak of the ERP components, were used: [1] 230 to 350 ms (ERAN), [2] 500 to $700 \mathrm{~ms}$ (N5), [3] 100 to 180 ms (early difference, mainly in the SLI group), and [4] 800 to $1000 \mathrm{~ms}$ (late difference, mainly in the SLI group). ${ }^{9}$ To guarantee for normality of the data, outliers were detected and removed (using the SPSS procedure EXAMINE). Thereafter, a Kolmogorov-Smirnov test revealed that the variables in the analyses did not deviate from a standard normal distribution $(0.383 \leq p \leq 0.990$; Median $=0.853)$.

The statistical analysis was performed in two steps: In a first step, the ERPs of both groups were statistically evaluated by a mixed-model ANOVA for repeated measures, containing the within-subject factors chord function (supertonics vs. tonics), anterior-posterior distribution, and hemisphere (left vs. right), as well as the between-subjects factor group (TLD vs. SLI). All results of these ANOVAs are summarized in Table 2, but only main effects and interactions with chord function (the experimentally manipulated factor) will be described in the Results section. Whenever the interaction of chord function $\times$ group was significant, two follow-up ANOVAs (with the same withinsubject factors as above) were computed, separately for each group of children. Within these ANOVAs, user-defined contrasts were employed to specify at which ROI the difference between the two chord functions was significant. For the ERAN, for which a previous study revealed gender differences in the laterlization (Koelsch et al., 2003), two further ANOVAs (separately for each group) with the factors chord function, hemisphere, and sex

9 Two - [1] ERAN and [2] N5 - were set to ERP components that are consistently elicited by violations of musical syntax (see, e.g., Koelsch et al., 2003; Koelsch et al., 2000). Two other time windows - [3] and [4] - were suggested by visual inspection of the ERPs. 
were computed (for the anterior ROIs, at which the ERAN is strongest).

Using linear regression analyses, we determined whether the socioeconomic background of the children influenced the amplitudes of the evaluated ERP components. Correlation analyses were used to investigate the relation between the ERP indicators of music-syntactic processing and the behavioural measures of linguistic abilities, non-verbal intelligence, and musical skills. Because of the bimodal distribution of the most variables (i.e., the relatively distinct values in the two groups) nonparametric (Spearman) correlations were used.

\section{Results}

To ensure that difficulties of children with SLI in their processing of musical syntax are not due to deficiencies in early stages of auditory processing, the ERP response to the onset of the first chord of the sequences was evaluated. The ERP response had a larger amplitude at anterior scalp sites and was essentially the same in both groups (see Figure 2), in the earlier $(0$ to $100 \mathrm{~ms}$, TLD: $M=2.93 \mu \mathrm{V}$, $S E M=0.34 \mu \mathrm{V} \quad$ vs. $\quad$ SLI: $M=2.96 \mu \mathrm{V}$, $S E M=0.39 \mu \mathrm{V}$; anterior ROIs) and the later time window $(100$ to $200 \mathrm{~ms}$, TLD: $M=3.92 \mu \mathrm{V}, \quad S E M=0.87 \mu \mathrm{V} \quad$ vs. $\quad$ SLI: $M=4.54 \mu \mathrm{V}, S E M=1.00 \mu \mathrm{V})$. Neither a main effect of group, nor interactions of group with hemisphere, anterior-posterior distribution, or time window was yielded. That is, children with SLI did not differ from children with TLD at these early auditory processing stages.

ERAN. An ERAN was elicited in the TLD group in response to the irregular supertonics compared to the regular tonics $(M=-2.17 \mu \mathrm{V}, S E M$ $=0.33 \mu \mathrm{V}$; anterior ROIs). The ERAN was most prominent over frontal leads (see Figure 3 and 4), and had two peaks (rather than one single peak). This double peak was due to two different subgroups within the TLD children, one with a shorter (around $252 \mathrm{~ms} ; N=13$ ), and one with a longer peak latency of the
ERAN (around $339 \mathrm{~ms} ; N=7$ ). However, the individual subjects usually showed one clear peak, and the peak latencies were not correlated with the age of the participants $(r=-0.26$; $\mathrm{p}=0.131$ ).

In contrast to the TLD group, no ERAN was elicited in the SLI group (nominally, irregular chords elicited even more positive amplitude values than regular ones, $M=0.82 \mu \mathrm{V}$, $S E M=0.77 \mu \mathrm{V}$, although this difference was statistically not significant, see below).

An ANOVA revealed an interaction of chord function $\times$ group and an interaction of chord function $\times$ anterior-posterior distribution $\times$ group (reflecting that the ERAN was most prominent over anterior sites in the group of children with TLD, see second column of Table 2 for detailed results). The main effect of group was not significant, suggesting that the two groups differed in their ERP response to the chord functions (supertonics vs. tonics) but were comparable in their overall amplitude values. Further ANOVAs conducted separately for each group revealed a main effect of chord function for the TLD group $\left(F_{(1,19)}=24.10 ; p<\right.$ $0.001)$, and an interaction of chord function $\times$ anterior-posterior distribution $\left(F_{(1,19)}=9.18 ; p=\right.$ $0.007)$. In this group, the difference in the ERP response to the two chord functions was significant at both anterior ROls (left: $F_{(1,19)}=$ $22.51 ; p<0.001$; right: $F_{(1,19)}=23.17 ; p<$ 0.001 ; tested with user-defined contrasts). For the SLI group, neither a main effect nor any interaction with chord function was found. That is, an ERAN was observed over anterior sites in the children with TLD but not in the children with SLI. A previous study suggested gender differences in the lateralization of the ERAN (Koelsch et al., 2003), but ANOVAs did not indicate a difference in lateralization of the ERAN between sexes (i.e., an interaction of chord function $\times$ hemisphere $\times$ sex), neither for the TLD group $\left(F_{(1,18)}=2.59 ; p=0.125\right)$, nor for the SLI group $\left(F_{(1,13)}=0.02 ; p=0.866\right)$.

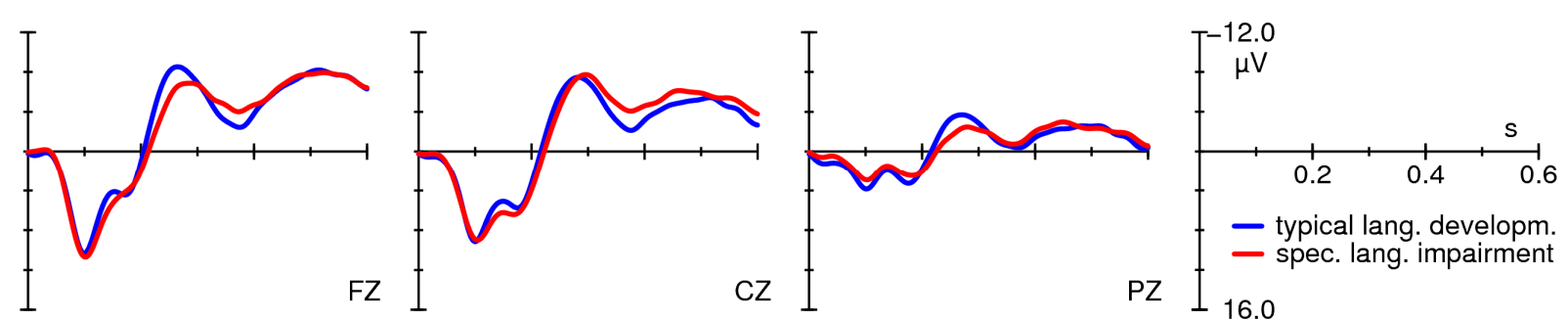

Figure 2. ERP responses to the onset of the first chord in the sequence: Children with typical language development (blue lines) were compared to children with specific language impairment (red lines). 


\section{Typical language development}

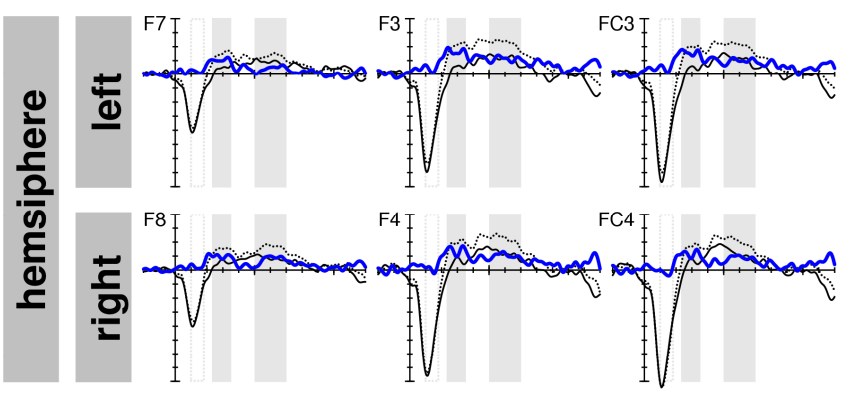

Comparison of the groups

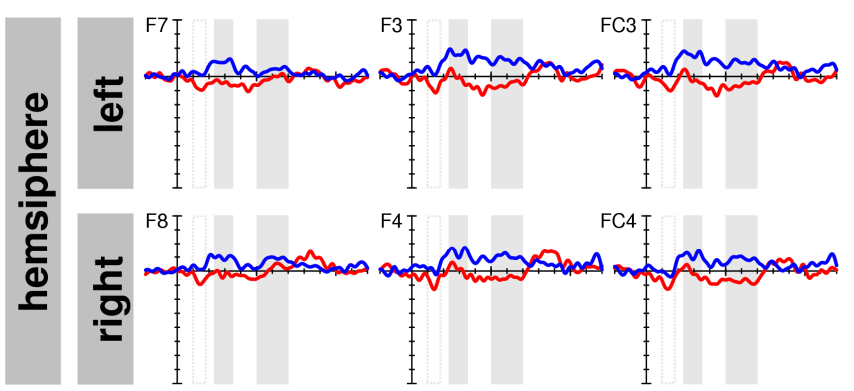

\section{Specific language impairment}

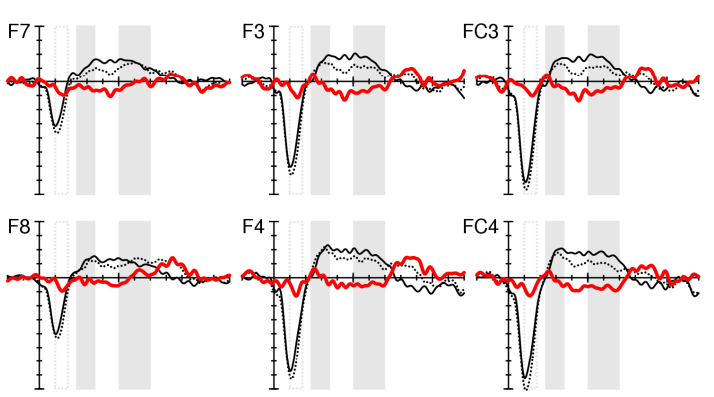

\section{Legend}
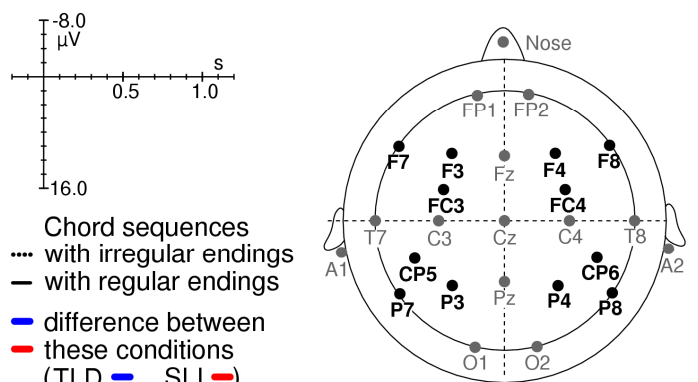

Figure 3. ERPs elicited at electrodes of anterior ROIs, separately for children with typical language development (TLD; top-left panel) and for children with specific language impairment (SLI; top-right panel). The rectangles indicate time windows used for statistical analysis: 100 - $180 \mathrm{~ms}$ (early acoustic processing, left rectangle), 230 $350 \mathrm{~ms}$ (ERAN, middle gray shaded area) and $500-700 \mathrm{~ms}$ (N5, right gray shaded area). Black dotted lines represent the brain responses to the irregular endings of the chord sequences, and black solid lines the responses to the regular endings. Blue (children with TLD) and red lines (children with SLI) indicate the differencewaveforms between conditions (irregular subtracted from regular ERPs). Direct comparison of the difference waveforms for both groups of children (TLD (blue) vs. SLI (red); bottom-left panel). Electrode positions are shown on the schematic head with electrodes of the ROIs marked in black (bottom-right panel).

chord function

chord function $\times$ group

chord function $\times$ ant.-post. $\times$ group

anterior-posterior distribution

ant.-post. $\times$ hemisphere

group

Table 2. Overview of the results from the ANOVAs Where investigated: $100-180 \mathrm{~ms}$ (early acoustic processing), $230-350 \mathrm{~ms}$ (ERAN) and $500-700 \mathrm{~ms}$ (N5). Only effects significant at least within one time window are reported in the table. Main effects and interactions with chord function are printed in the uppermost part of the table. Significant effects are written in black.

N5. In the children with TLD, an N5 was observed $(M=-1.47 \mu \mathrm{V}$, SEM $=0.52 \square \mathrm{V})$ which had an amplitude maximum around 500 to 600 $\mathrm{ms}$ and was most prominent at frontal electrodes (see Figure 3 and 4 ). In contrast, in the children with SLI, the difference between the two chord functions had a positive polarity $(M=$ $1.14 \mu \mathrm{V}, S E M=0.87 \mu \mathrm{V}$ ) and an amplitude maximum at lateral posterior sites (see Figure 4).

An ANOVA revealed a significant interaction of chord function $\times$ group, reflecting that an N5 was observed in the children with TLD, but not in those with SLI (see third column of Table 2 for detailed results). The main effect of group was not significant, indicating that the overall amplitude values were similar in both groups. 
ANOVAs computed separately for each group revealed for the TLD group a main effect of chord function $\left(F_{(1,19)}=5.45 ; p=0.031\right)$ as well as an interaction of chord function $\times$ anteriorposterior distribution $\left(F_{(1,19)}=6.15 ; p=0.023\right)$. The same results were obtained employing user-defined contrasts, namely, a significant amplitude difference at both anterior ROls (left: $F_{(1,19)}=4.49 ; p=0.048$; right: $F_{(1,19)}=8.90 ; p=$ $0.008)$. In the group of children with SLI, the ERP responses to the two chord functions did not differ significantly. That is, an N5 was found in the TLD group (with a distribution most prominent over the anterior ROIs) which could not be observed in children with SLI.

In addition to the results for ERAN and N5, which were in accordance with our hypotheses, there was a further, unexpected finding: ERP responses to tonics and supertonics differed in the children with SLI around 100 to $180 \mathrm{~ms}$ after stimulus onset (see Figure 3 ). In children with SLI, tonic chords elicited a less positive potential $(M=4.89 \mu \mathrm{V}, S E M=0.66 \square \mathrm{V}$; mean amplitude of all ROIs) compared to children with TLD $(M=6.65 \mu \mathrm{V}, S E M=0.57 \square \mathrm{V})$. The ERP responses to the supertonics were similar in both groups (SLI: $M=6.42 \mu \mathrm{V}$, $S E M=0.65 \square \mathrm{V} ;$ TLD: $M=6.67 \mu \mathrm{V}$, SEM $=0.57$ $\square \mathrm{V})$.

An ANOVA revealed a main effect of chord function, an interaction of chord function $x$ group, but no main effect of group (see first column of Table 2 for detailed results). That is, a difference in the ERP responses was observed, mainly in the children with SLI, whereas the amplitude values per se did not differ in the two groups. Two further ANOVAs, computed for each group separately, revealed a main effect of chord function in the group of children with SLI $\left(F_{(1,14)}=10.72, p=0.006\right)$. The difference in the response to the two chord functions was significant at all ROls except the right-posterior ROI (left-anterior: $F_{(1,14)}=5.58 ; p=0.033$; rightanterior: $F_{(1,14)}=5.70 ; p=0.032$; left-posterior: $F_{(1,14)}=5.84 ; \mathrm{p}=0.030$; employing user-defined contrasts). In the TLD group neither a main effect nor interactions with chord function were found.

Although ERP waveforms slightly differed in the time window around 800 to $1000 \mathrm{~ms}$, this difference was statistically not significant.

Regression and correlation analyses: Using a linear regression analysis, we determined whether the socioeconomic status (ISEI values of mothers and fathers), and the duration of parents' education influenced the processing of music-syntactic regularities. However, these variables were not suitable predictors, neither for the ERAN $(0.150 \leq p \leq 0.922)$ nor for the N5 $(0.164 \leq p \leq 0.965)$. This indicates that the observed group differences in the amplitudes of
ERAN and N5 were not due to differences in the social background. Furthermore, we hypothesized that children with SLI would have difficulties in their syntax processing for music and language. To test this assumption, we computed correlations between the ERAN and the N5 amplitude (at anterior ROls; rows of Table $3)^{10}$ and the subtests of the language development test, the tests of musical abilities, and the subtests of the non-verbal intelligence test (columns of Table 3). ${ }^{11}$

All four subtests of the language development test were correlated with the ERAN amplitude (see first columns of Table 3). These subtests reflect the complex interplay of phonological, lexical-semantic, and linguistic syntax processing. Furthermore, most of these tasks require intact attention and working memory functions, which are important for establishing structural relations during on-line processing. The task "Non-word repetition" is regarded as a prominent marker of phonological working memory functions.

In addition, several tests of musical abilities were conducted. Within these, only the factor representing "Memory for musical phrases" (see middle column in Table 3 ) had a significant relationship with the ERAN amplitude. This factor represents how fast the children were able to differentiate between the original or a modified versions of a musical phrase (in a paired comparison). It requires melodic and rhythmicmelodic processing, both being central aspects of music-syntactic processing. The correlation also underlines the importance of short-term memory functions (i.e. the ability to represent musical phrases) for music-syntactic processing.

10 For the amplitude of the early difference (around 100 to $180 \mathrm{~ms}$ ) no significant correlations were found.

11 Most correlations are negative since superior results in the tests (mostly for children with TLD) are related to large (more negative) amplitudes of the ERAN and the N5 whereas inferior results in the tests (mostly for children with SLI) are linked to small (less negative) amplitudes. For "Musical memory" the correlation is positive as shorter reaction times and a larger ERAN and N5 amplitude were observed in the children with TLD and longer reaction times and smaller amplitudes in children with SLI. 


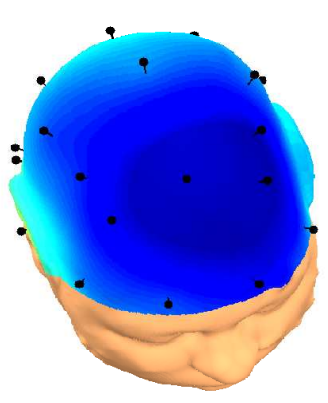

$230-350 \mathrm{~ms}$ (ERAN)

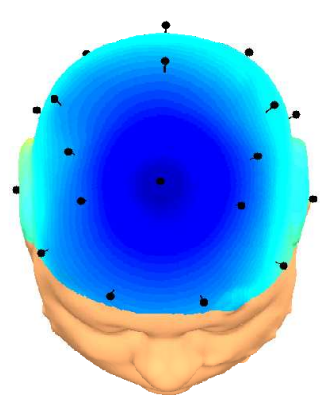

$500-700 \mathrm{~ms}$ (N5)

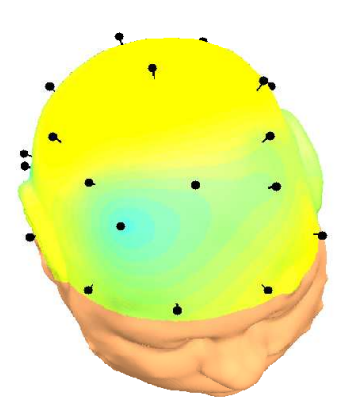

230 - $350 \mathrm{~ms}$ (ERAN)

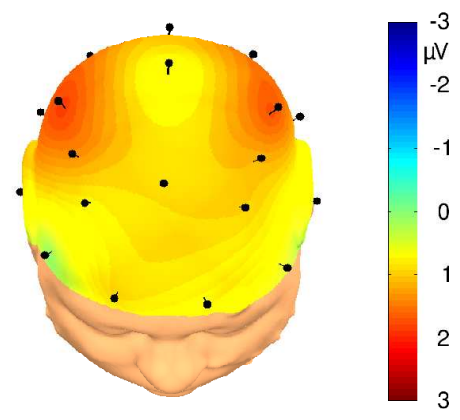

$500-700 \mathrm{~ms}(\mathrm{~N} 5)$

Figure 4. Scalp topography of the difference ERPs (irregular subtracted from regular final chords), separately for children with typical language development (left panel) and children with specific language impairment (right panel). Time windows for interpolation of ERPs were 230 - $350 \mathrm{~ms}$ (ERAN, left in each panel), and 500 $700 \mathrm{~ms}$ (N5, right in each panel)

\begin{tabular}{lrrrrrrrrr}
\hline & & $\begin{array}{r}\text { sentence } \\
\text { compreh. }\end{array}$ & $\begin{array}{r}\text { plural } \\
\text { generat. }\end{array}$ & non-word & sentence & musical & hand & triangles & $\begin{array}{r}\text { spatial } \\
\text { memory }\end{array}$ \\
& $N$ & 35 & 35 & 35 & 35 & 25 & 35 & 35 & 29 \\
ERAN & $r$ & -0.390 & -0.377 & -0.456 & -0.419 & 0.428 & -0.397 & -0.239 & -0.452 \\
N5 & $p$ & 0.021 & 0.026 & 0.006 & 0.012 & 0.037 & 0.020 & 0.174 & 0.014 \\
\cline { 2 - 9 } & $r$ & -0.093 & -0.295 & -0.293 & -0.262 & 0.296 & -0.338 & -0.352 & -0.462 \\
& $p$ & 0.597 & 0.085 & 0.087 & 0.129 & 0.161 & 0.051 & 0.041 & 0.012 \\
\hline
\end{tabular}

Table 3. Correlations $(r)$ and their statistical significance $(p)$ for the amplitudes of the ERAN, and the N5 (mean of the anterior ROIs) with measures of linguistic abilities, non-verbal intelligence, and musical abilities ( $N$ : number of participants in that test). Significant correlations are written in black.

Some theoretical accounts to SLI assume general processing limitations which may be reflected in a lowered general intelligence. Thus, we computed correlations of the amplitudes of ERAN and N5 with the subtests of a non-verbal intelligence test (see last columns in Table 3). The subtests "Hand movements" and "Spatial memory" correlated significantly with the amplitude of the ERAN. For the N5 amplitude, correlations were found with the subtests "Triangles" and "Spatial memory". This strengthens the assumptions of the importance of working memory functions and the ability to process and store ordered sequences. These skills are crucial for these tests as well as for the processing of musical and linguistic syntax.

\section{Discussion}

Our study compared the processing of musical syntax in children with TLD and with SLI. In 4-to-5-year old children with TLD, an ERAN and an N5 were observed. These ERP components are comparable to those elicited by musicsyntactic violations in adults (e.g., Koelsch et al., 2000; Koelsch et al., 2007). In children, the latency of the ERAN was longer (replicating findings of an earlier study; Koelsch et al., 2003), and had a larger variance. The latencies were not correlated with the age of the participants, suggesting that the increased variance was rather not due to different environmental stages. Notably, the final supertonics represented a music-syntactic, but not a physical irregularity (see also Methods). Our findings that these chords elicit both ERAN and N5 thus demonstrate that already 4-to-5-year old children possess cognitive representations of the syntactic regularities of Western tonal music, and that they process music fast and accurately according to these representations.

In contrast, neither ERAN nor N5 were elicited in children with SLI, showing that SLI children clearly differ from TLD children in their processing of music-syntactic information. However, children with TLD and SLI did not differ in their processing of acoustic features: no difference between the two groups was observed in the ERP response to the onset of the first chord of the sequence. Moreover, even though the two groups differed with regard to the socioeconomic status of their parents, the amplitudes of the investigated ERP components were not influenced by these variables. Thus, it is likely 
that these variables did not account for the impaired processing of musical syntax in children with SLI. Their deficiencies in musicsyntactic processing appear comparable to their deficiencies in the processing of linguistic syntax. This provides further evidence that musical and linguistic syntax are processed in shared neural systems.

The observed, characteristic pattern of ERP responses (with the presence of ERAN and N5 in children with TLD and their absence in the children with SLI) strengthens the assumption of a strong relation of syntax processing in music and language: Deficiencies in musicsyntactic processing might be mirrored in comparable difficulties in linguistic-syntactic processing (in children with SLI). Conversely, children with TLD had neither difficulty in the language nor in the music domain. Moreover, because music-syntactic processing is already established in 21/2-year-olds (Jentschke, 2007), the presence or absence of the neurophysiological correlates of these processes might help to identify children at-risk to develop SLI.

An unexpected finding was the difference in the ERP responses to the two chord functions around 100 to $180 \mathrm{~ms}$ in the children with SLI, but not in the children with TLD. One possible explanation for this difference is that it reflects processes of the auditory sensory memory: As illustrated in Figure 1D, tonics (compared to supertonics) are acoustically slightly less congruent with the sensory memory traces generated by the preceding chords (see Figure 1D, and Koelsch et al., 2007 for discussion). Due to this slight acoustic deviance, final tonics might have elicited a mismatch negativity (MMN; Näätänen, Schröger, Karakas, Tervaniemi, \& Paavilainen, 1993). In children with TLD, this MMN response would have been masked by the ERAN elicited by the supertonics, whereas this MMN was still visible in SLI children, because no ERAN was elicited in this group. However, future studies should replicate, and further investigate this effect.

The assumption of shared neural networks for music- and linguistic-syntactic processing is strengthened by the correlations of the ERAN amplitude with behavioural measures, especially from those with the subtests of the language development test. Prerequisite for these subtests is sophisticated grammar processing, that is, knowledge of grammatical structures and the ability to extract relationships of elements in the perceived sequence. Thus, the difficulties of the SLI children are clearly related to their processing deficits for structural dependencies in music and language.

Another requirement for the processing of musical or sentential phrases is working memory which is necessary to hold the elements of a phrase in memory, to group these elements together (to form a coherent percept) and to build relations between these elements (to extract the underlying structure). These functions may be impaired in children with SLI (Gathercole \& Alloway, 2006), contributing to their deficiencies in both, processing of musical and linguistic syntax. The correlations of the ERAN amplitude with the subtests "Spatial Memory" and "Hand Movement" emphasize the necessity for encoding and storing information in short-term memory and the ability to process and store ordered sequences. Bauer, Hertsgaard, Dropik, and Daly (1998) demonstrated that the accurate reproduction of ordered (event) sequences was predictive for later language development. The correlation with "Memory for musical phrases" further strengthens the view of the importance of working memory functions because these subtests build strongly upon such working memory. In contrast for musical skills, which are based on long-term memory no correlation with the ERP measures was observed,. Syntax allows cognitive "chunking" and thus makes it easier to process and to remember long complex sequences (Simon, 1962, 1972). Therefore, deficiencies in syntax processing may lead to a decreased in working memory performance. Vice versa, decreased working memory capacity may contribute to the deficiencies in the processing of musical and linguistic syntax in children with SLI, because intact working memory functions may be a prerequisite for extracting the structural relationships between the elements of a sequence.

Our results demonstrate that in children with SLI, deficiencies in the language domain are mirrored in comparable difficulties in the music domain. Conversely, in children with TLD neither difficulties in music-syntactic nor in linguistic-syntactic processing were observed. This pattern may result from overlapping neuronal networks that underlie the processing of musical and linguistic syntax. Functionally, the deficiencies in children with SLI may be based on a common underlying factor, which is related to the processing and storing of ordered sequences that are organized according to surface and regularities and underlying structures. Deficiencies in the processing of ordered sequences may be related to an impaired procedural memory system in children with SLI (Ullman \& Pierpont, 2005). In addition, some prerequisite abilities (such as working memory functions) that are essential for syntax processing in both domains may also be impaired in children with SLI.

The interpretation put forward here is in line with the view that music and speech are intimately connected in early life (Trehub, 2003), and that music paves the way to linguistic capacities (Fernald, 1989; Papoušek, 1996). Under such a view it seems possible that music 
perception might implicitly train parts of the language network and, thus, be an important contribution to the treatment of children with SLI. Our results might also stimulate future research to identify children at risk for SLI at younger ages. In such children, musical training might even prevent from the development of SLI.

\section{Conclusion}

The present study indicates that processing of musical syntax elicits an ERP pattern in children with TLD which is comparable to older children and adults. In children with SLI, however, a different ERP pattern was found, reflecting their difficulties to process music-syntactic regularities. Correlations of the ERAN amplitude with measures of linguistic and musical abilities provide further indications for the strong relationship of syntax processing in music and language and point to similar difficulties of children with SLI in both domains. This relationship is in agreement with previous evidence of comparable cognitive mechanisms and shared underlying neural resources for the processing of linguistic and musical information. A better understanding of the neural mechanisms underlying this relationship - as provided by our results - opens a new perspective for more a effective treatment for language impaired children that includes musical training. Such training might perhaps even prevent from the development of SLI, particularly in children at risk for the development of SLI.

\section{Acknowledgments}

We thank our participants and their parents, the teachers in the kindergartens who helped to recruit our participants, and the kindergarten for special education for the opportunity to run our EEG measurements there. Ulrike Barth and Kristiane Werrmann helped to acquire the data. Benedicte Poulin-Charronat, Daniela Sammler, and Daniel Mietchen gave valuable comments, improving the manuscript. This work was supported by a grant of the German Research Foundation awarded to S.K. (KO 2266/2-1/2).

\section{References}

Alcock, K. J., Passingham, R. E., Watkins, K., \& Vargha-Khadem, F. (2000). Pitch and timing abilities in inherited speech and language impairment. Brain and Language, 75(1), 34-46.

American Electroencephalographic Society. (1994). Guideline 13: Guidelines for standard electrode position nomenclature. Journal of Clinical Neurophysiology, 11(1), 111-113.

Anvari, S. H., Trainor, L. J., Woodside, J., \& Levy, B. A. (2002). Relations among musical skills, phonological processing, and early reading ability in preschool children. Journal of Experimental Child Psychology, 83(2), 111130.

Bauer, P. J., Hertsgaard, L. A., Dropik, P., \& Daly, B. P. (1998). When even arbitrary order becomes important: developments in reliable temporal sequencing of arbitrarily ordered events. Memory, 6(2), 165-198.

Bishop, D. V. M. (2004). Diagnostic dilemmas in specific language impairment. In L. Verhoeven \& $\mathrm{H}$. van Balkom (Eds.), Classification of developmental language disorders (pp. 309326). Mahwah, NJ: Lawrence Erlbaum.

Bishop, D. V. M., \& McArthur, G. M. (2004). Immature cortical responses to auditory stimuli in specific language impairment: evidence from ERPs to rapid tone sequences. Developmental Science, 7(4), F11-18.

Botting, N. (2005). Non-verbal cognitive development and language impairment. Journal of Child Psychology and Psychiatry and Allied Disciplines, 46(3), 317-326.

Botting, N., Faragher, B., Simkin, Z., Knox, E., \& Conti-Ramsden, G. (2001). Predicting pathways of specific language impairment: what differentiates good and poor outcome? Journal of Child Psychology and Psychiatry and Allied Disciplines, 42(8), 1013-1020.

Delorme, A., \& Makeig, S. (2004). EEGLAB: an open source toolbox for analysis of single-trial EEG dynamics including independent component analysis. Journal of Neuroscience Methods, 134(1), 9-21.

Deutsch, D. (1999). The psychology of music (2nd ed.). San Diego: Academic Press.

Fernald, A. (1989). Intonation and communicative intent in mothers' speech to infants: is the melody the message? Child Development, 60(6), 1497-1510.

Ganzeboom, H. B. G., \& Treiman, D. J. (1996). Internationally Comparable Measures of Occupational Status for the 1988 International Standard Classification of Occupations. Social Science Research, 25(3), 201-239.

Gathercole, S. E., \& Alloway, T. P. (2006). Practitioner review: short-term and working memory impairments in neurodevelopmental disorders: diagnosis and remedial support. Journal of Child Psychology and Psychiatry and Allied Disciplines, 47(1), 4-15.

Grimm, H., Aktas, M., \& Frevert, S. (2001). SETK 3-5: Sprachentwicklungstest für dreibis fünfjährige Kinder [Language development test for three to five year old children; german version]. Göttingen: Hogrefe.

International Labour Organization. (1990). ISCO88. International Standard Classification of Occupations. Geneve, $\mathrm{CH}$ : International Labour Office.

Jentschke, S. (2007). Neural Correlates of Processing Syntax in Music and Language Influences of Development, Musical Training, 
and Language Impairment. Unpublished PhD thesis, University of Leipzig, Leipzig, Germany.

Jusczyk, P. W. (2002). How Infants Adapt Speech-Processing Capacities to NativeLanguage Structure. Current Directions in Psychological Science, 11(1), 15-18.

Jusczyk, P. W., Hirsh-Pasek, K., Nelson, D. G., Kennedy, L. J., Woodward, A., \& Piwoz, J. (1992). Perception of acoustic correlates of major phrasal units by young infants. Cognitive Psychology, 24(2), 252-293.

Kaufman, A. S., Kaufman, N. L., \& Melchers, P. (1994). K-ABC: Kaufman Assessment Battery for Children (German ed.). Amsterdam: Swets \& Zeitlinger.

Koelsch, S. (2005). Neural substrates of processing syntax and semantics in music. Current Opinion in Neurobiology, 15(2), 207-212.

Koelsch, S., Grossmann, T., Gunter, T. C., Hahne, A., Schröger, E., \& Friederici, A. D. (2003). Children processing music: electric brain responses reveal musical competence and gender differences. Journal of Cognitive Neuroscience, 15(5), 683-693.

Koelsch, S., Gunter, T., Friederici, A. D., \& Schröger, E. (2000). Brain indices of music processing: "nonmusicians" are musical. Journal of Cognitive Neuroscience, 12(3), 520-541.

Koelsch, S., Gunter, T. C., Schröger, E., Tervaniemi, M., Sammler, D., \& Friederici, A. D. (2001). Differentiating ERAN and MMN: an ERP study. Neuroreport, 12(7), 1385-1389.

Koelsch, S., Gunter, T. C., Wittfoth, M., \& Sammler, D. (2005). Interaction between syntax processing in language and in music: an ERP Study. Journal of Cognitive Neuroscience, 17(10), 1565-1577.

Koelsch, S., Jentschke, S., Sammler, D., \& Mietchen, D. (2007). Untangling syntactic and sensory processing: An ERP study of music perception. Psychophysiology, 44(3), 476490.

Koelsch, S., \& Mulder, J. (2002). Electric brain responses to inappropriate harmonies during listening to expressive music. Clinical Neurophysiology, 113(6), 862-869.

Koelsch, S., Schmidt, B. H., \& Kansok, J. (2002). Effects of musical expertise on the early right anterior negativity: an eventrelated brain potential study. Psychophysiology, 39(5), 657-663.

Koelsch, S., Schröger, E., \& Gunter, T. C. (2002). Music matters: preattentive musicality of the human brain. Psychophysiology, 39(1), 38-48.

Krumhansl, C. L., \& Jusczyk, P. W. (1990). Infants' perception of phrase structure in music. Psychological Science, 1(1), 70-73.

Leino, S., Brattico, E., Tervaniemi, M., \& Vuust, P. (2007). Representation of harmony rules in the human brain: further evidence from eventrelated potentials. Brain Research, 1142, 169177.

Leman, M., Lesaffre, M., \& Tanghe, K. (2005). IPEM Toolbox for Perception-Based Music Analysis (Version 1.02). from http://www.ipem.ugent.be/Toolbox/index.html

Leonard, L. B. (1998). Children with specific language impairment. Cambridge, MA: MIT Press.

Leonard, L. B. (2000). Specific language impairment across languages. In D. V. M. Bishop \& L. B. Leonard (Eds.), Speech and language impairments in children: Causes, characteristics, intervention and outcome (pp. 115-130). Hove: Psychology Press.

Lerdahl, F. (2001). Tonal pitch space. New York: Oxford University Press.

Loui, P., Grent-'t-Jong, T., Torpey, D., \& Woldorff, M. (2005). Effects of attention on the neural processing of harmonic syntax in Western music. Brain Research. Cognitive Brain Research, 25(3), 678-687.

Magne, C., Schön, D., \& Besson, M. (2006). Musician children detect pitch violations in both music and language better than nonmusician children: behavioral and electrophysiological approaches. Journal of Cognitive Neuroscience, 18(2), 199-211.

Marinellie, S. A. (2004). Complex syntax used by school-age children with specific language impairment (SLI) in child-adult conversation. Journal of Communication Disorders, 37(6), 517-533.

McArthur, G. M., \& Bishop, D. V. M. (2004). Frequency discrimination deficits in people with specific language impairment: reliability, validity, and linguistic correlates. Journal of Speech, Language, and Hearing Research, 47(3), 527-541.

McArthur, G. M., \& Bishop, D. V. M. (2005). Speech and non-speech processing in people with specific language impairment: a behavioural and electrophysiological study. Brain and Language, 94(3), 260-273.

McMullen, E., \& Saffran, J. R. (2004). Music and Language: A Developmental Comparison. Music Perception, 21(3), 289-311.

Näätänen, R., Schröger, E., Karakas, S., Tervaniemi, M., \& Paavilainen, P. (1993). Development of a memory trace for a complex sound in the human brain. Neuroreport, 4(5), 503-506.

Overy, K., Nicolson, R. I., Fawcett, A. J., \& Clarke, E. F. (2003). Dyslexia and music: measuring musical timing skills. Dyslexia, 9(1), 18-36.

Papoušek, H. (1996). Musicality in infancy research. In J. A. Sloboda \& I. Deliege (Eds.), Musical Beginnings. Oxford: Oxford University Press. 
Patel, A. D. (2003). Language, music, syntax and the brain. Nature Neuroscience, 6(7), 674-681.

Patel, A. D., Gibson, E., Ratner, J., Besson, M., \& Holcomb, P. J. (1998). Processing syntactic relations in language and music: an eventrelated potential study. Journal of Cognitive Neuroscience, 10(6), 717-733.

Riemann, H. (1877). Musikalische Syntaxis. Grundriss einer harmonischen Satzbildungslehre. Leipzig: Breitkopf und Härtel.

Schön, D., Magne, C., \& Besson, M. (2004). The music of speech: music training facilitates pitch processing in both music and language. Psychophysiology, 41(3), 341-349.

Simon, H. A. (1962). The Architecture of Complexity. Proceedings of the American Philosophical Society, 106(6), 467-482.

Simon, H. A. (1972). Complexity and the representation of patterned sequences of symbols. Psychological Review, 79(5), 369-382.

Slevc, L. R., \& Miyake, A. (2006). Individual differences in second-language proficiency: does musical ability matter? Psychological Science, 17(8), 675-681.

Slevc, L. R., Rosenberg, J. C., \& Patel, A. D. (2007). Making Psycholinguistics Musical: Evidence for shared processing of linguistic and musical syntax. Paper presented at the Conference "Language and Music as Cogni- tive Systems", May 11-13, 2007, Cambridge, UK.

Steinbeis, N., Koelsch, S., \& Sloboda, J. A. (2006). The role of harmonic expectancy violations in musical emotions: evidence from subjective, physiological, and neural responses. Journal of Cognitive Neuroscience, 18(8), 1380-1393.

Tillmann, B., Bharucha, J. J., \& Bigand, E. (2000). Implicit learning of tonality: a selforganizing approach. Psychological Review, 107(4), 885-913.

Trehub, S. E. (2003). Musical predispositions in infancy: An update. In I. Peretz \& R. J. Zatorre (Eds.), The cognitive neuroscience of music (pp. 3-20). Oxford: Oxford University Press.

Ullman, M. T., \& Pierpont, E. I. (2005). Specific language impairment is not specific to language: the procedural deficit hypothesis. Cortex, 41(3), 399-433.

van der Lely, H. K. J. (2005). Domain-specific cognitive systems: insight from GrammaticalSLI. Trends in Cognitive Sciences, 9(2), 5359.

Wong, P. C., Skoe, E., Russo, N. M., Dees, T., \& Kraus, N. (2007). Musical experience shapes human brainstem encoding of linguistic pitch patterns. Nature Neuroscience, 10(4), 420-422. 